\title{
STABILITY ANALYSIS AND CONTROL OF A 3-D AUTONOMOUS AI-YUAN-ZHI-HAO HYPERCHAOTIC SYSTEM
}

\author{
Edwin A. Umoh \\ Department of Electrical Engineering Technology \\ Federal Polytechnic, Zamfara State, NIGERIA \\ eddyumohegmail.com
}

\begin{abstract}
The stability analysis and control of a three-dimensional autonomous chaotic system that exhibits hyperchaotic properties is presented in this paper. The system's algebraic structure consists of six variable control parameters, three nonlinear terms and two cross-products which triggers hypersensitivity in its dynamic state evolutions. Stability analysis and control via fuzzy modelling confirms the controllability of its unstable orbits in well-tuned regions of stability using Lyapunov principles.
\end{abstract}

\section{KEYWORDS}

Chaos, fuzzy model, Lyapunov stability, hyperchaos

\section{INTRODUCTION}

Interest in the control of chaotic systems continues to attract attention among researchers even as their usefulness in modelling real systems continue to increase. The application of chaotic dynamics in complex engineering systems has motivated the search for more chaotic systems to match the complexities of these new challenges. In recent times, chaos has found applications in various engineering and non-engineering designs such as in medical science [1], psychology [2], economics and finance [3] and so on. Recently, chaos has been integrated into robotic systems to evolve new motion paradigm [4] for applicability of the robots in real world tasks such as firefighting devices [5], military operations [6]. In secure communications, chaos has found application in signal masking [7]. For a system to be chaotic, the following conditions must be satisfied. Firstly, it must be sensitive to perturbation in its initial conditions which should lead to unpredictability of its future trajectories, secondly, it must be topologically mixing and thirdly, the chaotic orbit must be dense [8]. Among some evolved chaotic attractors in the literature are the Chen's [9], 3-D, 4-wing attractor [10], Sundarapandian-Pehlivan [11], morphous oneparameter attractor [12], Rabinovich system [13]. When chaotic attractors possess one positive Lyapunov exponent, it is termed a basic chaotic system. However, when the system possesses two positive Lyapunov exponents it is a hyperchaotic system and becomes hypersensitive to small perturbations in its system dynamics. Control of chaotic system has become a fascinating subject of research because controllability and synchronizability of chaotic attractors are index of usefulness in a variety of designs such as in secure communications and robotics [14][15]. In this connection, various control strategies have been employed to stabilize chaotic regimes of 
attractors such backstepping [16], adaptive control [17], fuzzy control [18], passive control [19] and impulsive control [20]. In the context of stability and stabilization, the Lyapunov stability principles continued to enjoy wide usage and applications it can effectively stability issues in dissipative systems.

In this paper, analysis and synthesis of a fuzzy controller designed to control the chaotic regimes of a three-dimensional autonomous chaotic system that exhibits hyperchaotic properties is reported. The conditions for asymptotic stabilization of each fuzzy rule are also outlined.

\section{THE 3-D AI-YUAN-ZHI-HAO HYPERCHAOTIC SYSTEM}

The chaotic attractor under study has been proposed in [21]. The hyperchaotic system is made up of six control parameters and three nonlinear terms in the right equations. The system is represented by the following equations

$$
\begin{aligned}
& \frac{\partial x}{\partial t}=\psi(y-x)+\Omega y z \\
& \frac{\partial y}{\partial t}=\gamma x-\rho x z \\
& \frac{\partial y}{\partial t}=-\varphi z+\beta x^{2}
\end{aligned}
$$

Where $x, y, z$ are state variables and $\psi, \gamma, \rho, \varphi$ and $\beta$ are control parameters. For values of $\psi=10, \quad=25, \gamma=20, \rho=7, \varphi=8$ and $\beta=3$, the system (1) exhibits the chaotic attractor depicted in 2-D phase planes of Figure 1.

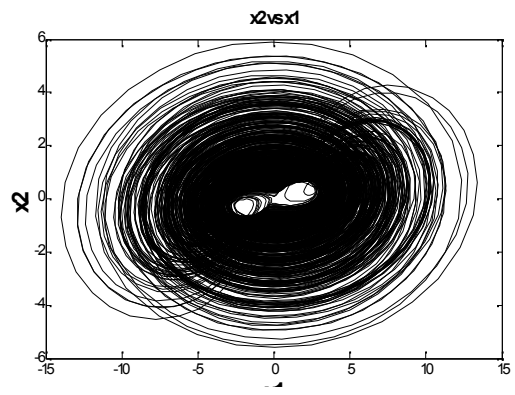

(a)

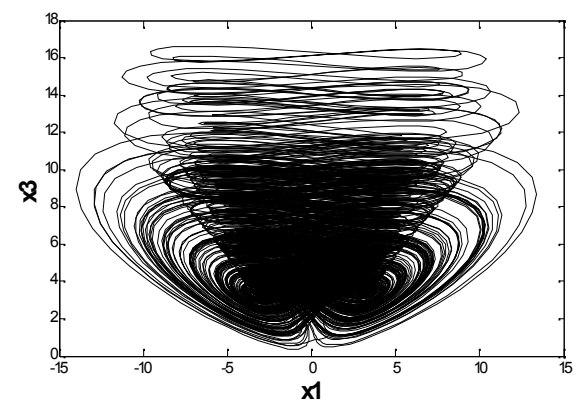

(b) 


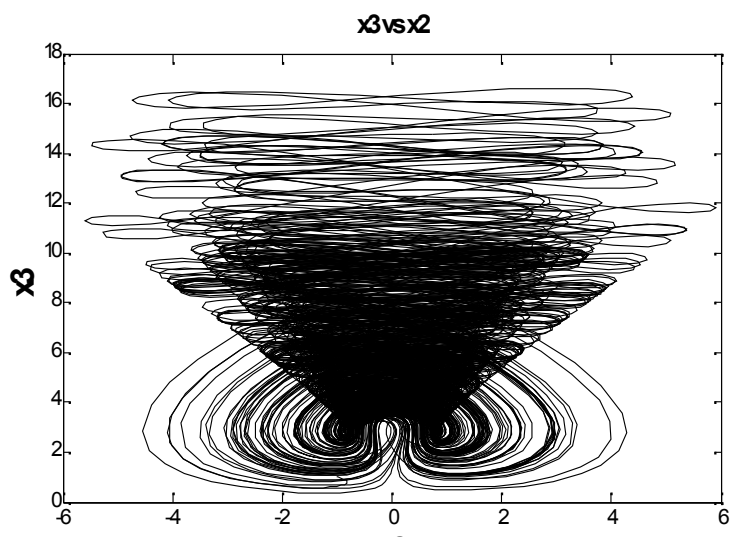

(c)

Figure 1. Evolution of system states in 2-D phase plane

The qualitative and quantitative properties of the attractor have been lucidly elaborated in [].

\section{FUZZY LOGIC CONTROLLER DESIGN AND LYAPUNOV BASED SYNTHESIS}

Chaotic system modelling based on fuzzy logic has long been practiced due to its to model chaotic dynamic systems due to its capability to approximate chaotic dynamics [22] . Of all fuzzy models, the Takagi-Sugeno (TS) fuzzy model [23] has played a dominant role in the modeling of complex nonlinear systems that are deficient of exact descriptions and have mathematically intractable dynamics. In the context of design approach, TS fuzzy modelling has relied on the linear matrix inequality (LMI) approach or the direct Lyapunov based analysis and synthesis. Although successful results based on LMI are replete in the literature, the stability analysis and synthesis often involves rigorous mathematical proofs [24]. On the other hand, the Lyapunov function based analysis and synthesis requires the selection of a Lyapunov function candidate whose partial derivative in continuous time would be render negative definite or negative semidefinite by a specified feedback control law [25].

\subsection{T-S Fuzzy controller design and synthesis}

A heuristic design methodology for a fuzzy controller that can guarantee stability was reported in [26]. In this approach, a fuzzy subsystem is formed by each fuzzy rule of the controller and the choice and distribution of membership function is not restrictive.

Definition 1 [27]: For any input $x_{0} \in W$, ( $W$ is the universe of discourse), if the degree of membership $\theta_{i}\left(x_{0}\right)$ corresponding to a fuzzy rule $i$ is zero, this fuzzy rule $\mathrm{i}$ is called an inactive fuzzy rule, otherwise it is called an active fuzzy rule.

In the active fuzzy region, the membership functions $\theta_{i}\left(x_{0}\right) \neq 0$, for all $x_{0} \in W_{r}$ where $W_{r} \subset W$ is the active region of the fuzzy rule. For proof (see [26]). 


\subsection{Design of the TS fuzzy controller}

Consider an autonomous nonlinear system comprising a plant and a Fuzzy Controller [27] described by

$$
\dot{x}=g(x)+k(x) u, x\left(t_{0}\right)=x_{0}
$$

Where $x=\left[x_{1}, x_{2}, \ldots \ldots x_{n}\right]^{\mathrm{T}}$ is a state vector, $g(x)=\left[g_{1}(x), g_{2}(x) \ldots \ldots g_{n}(x)\right]^{\mathrm{T}}$ and $k(x)=$ $\left[k_{1}(x), k_{2}(x) \ldots \ldots . k_{n}(x)\right]^{\mathrm{T}}$ are the function vectors related to the dynamics of the plant, $u$ is a control signal produced by the Fuzzy Controller. The Fuzzy Controller is made up of $n$ rules. The aggregate control signal applied to the dynamic system is a function of $u_{i}$ and $\varphi_{i}$ where $u_{i}$ is the control signal produced by each fuzzy subsystem that is formed by the fuzzy rules. The $i-t h$ fuzzy rule of the Fuzzy Controller is in the form of (3):

Rule $\boldsymbol{i}:$ IF $x_{1}$ is $\Phi_{i 1} A N D x_{2}$ is $\Phi_{i 2} A N D \ldots . . A N D x_{n}$ is $\Phi_{i n}$, THEN $u=u_{i}(x)$

Where $\Phi_{i 1}, X_{i 2} \ldots \Phi_{i n}$, are inputs fuzzy labels, and $u=u_{i}(x)$ is the control output.. Each fuzzy rule generates a degree of fulfillment $\theta_{i}(x)$ given by:

$$
\theta_{i}=\min \left(\theta_{i 1}, \theta_{i 2} \ldots \theta_{i n}\right) ; \quad \theta_{i} \in[0,1], i=1,2 \ldots n
$$

Definition 2 [27]: A fuzzy subsystem having a fuzzy rule $i$ is a system with a plant of (2) and controlled by only $u_{i}$, which is the output of fuzzy rule $i$ in the form of (3). By using the singleton- type fuzzifier with min-max inference type and the weighted sum defuzzification method, the aggregate Fuzzy Controller output control signal applied to the plant is related by equation (5):

$$
U=\frac{\sum_{i=1}^{n} \theta_{i}(x) u_{i}(x)}{\sum_{i=1}^{n} \theta_{i}(x)}
$$

The control scheme, triangular membership function and the fuzzy rule table are depicted below.

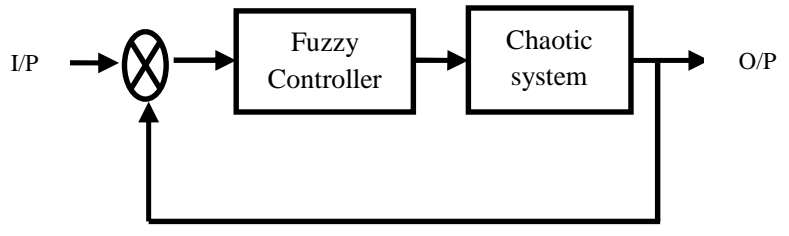

Figure 2. Fuzzy logic control scheme 
International Journal on Soft Computing, Artificial Intelligence and Applications (IJSCAI), Vol.2, No.4, August 2013
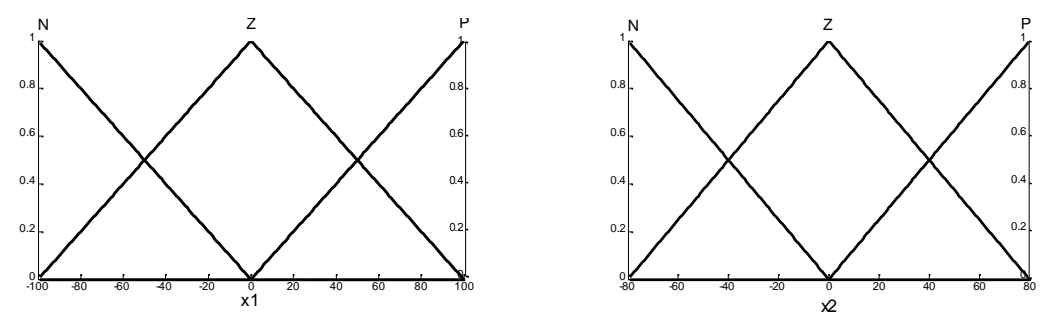

Figure 3: Triangular membership function for $x_{1}, x_{2} ; x_{1} \in[-100100] ; x_{2} \in[-8080] ; x_{3} \in \varnothing$

Table 1. Fuzzy rule base

\begin{tabular}{|c|c|c|c|}
\cline { 2 - 4 } \multicolumn{1}{c|}{} & $\mathbf{N}$ & $\mathbf{Z}$ & $\mathbf{P}$ \\
\hline $\mathbf{N}$ & $\mathrm{u}_{2}$ & $\mathrm{u}_{6}$ & $\mathrm{u}_{4}$ \\
\hline $\mathbf{Z}$ & $\mathrm{u}_{8}$ & $\mathrm{u}_{9}$ & $\mathrm{u}_{7}$ \\
\hline $\mathbf{P}$ & $\mathrm{u}_{3}$ & $\mathrm{u}_{5}$ & $\mathrm{u}_{1}$ \\
\hline
\end{tabular}

\subsection{Stabilizing controller synthesis}

In this section, we choose a Lyapunov function candidate $V(x, y, z): R^{n} \rightarrow R$ such that $V(0,0,0)=$ $0 ; V(x, y, z)>0 \forall(x, y, z) \neq 0 . V(x, y, z)$ is a continuous real-value function which also satisfies the following conditions:

$$
V(x, y, z, t) \leq 0 \forall(x, y, z) \neq 0 .
$$

The controlled system of (1) is derived by adding a control variable $u$ to the first equation such that

$$
\begin{aligned}
& \frac{\partial x}{\partial t}=\psi(y-x)+\Omega y z+u \\
& \frac{\partial y}{\partial t}=\gamma x-\rho x z \\
& \frac{\partial y}{\partial t}=-\varphi z+\beta x^{2}
\end{aligned}
$$

We choose a Lyapunov function candidate:

$$
V^{j}(x, y, z)=\frac{1}{2}\left(2 x^{2}+y^{2}+z^{2}\right), \quad j=1,2 \ldots n, n=9
$$

( $\mathrm{n}$ is the number of fuzzy rules). The partial derivative of (8) is given as

$$
\dot{V}^{j}(x, y, z)=2 x \dot{x}+y \dot{y}+z \dot{z}
$$

Putting (7) into (8) and simplifying: 
International Journal on Soft Computing, Artificial Intelligence and Applications (IJSCAI), Vol.2, No.4, August 2013

$$
\begin{gathered}
\dot{V}^{j}(x, y, z)=2 x(\psi(y-x)+\Omega y z+u)+y(\gamma x-\rho x z)+z\left(-\varphi z+\beta x^{2}\right) \\
=2 \psi x y-2 \psi x^{2}+\Omega x y z+2 x u+\gamma x y-\rho x y z-\varphi z^{2}+\beta x^{2} z
\end{gathered}
$$

State variable is assumed to be an empty set, i.e $z=0$ [27], therefore,

$$
\dot{V}^{j}(x, y, z, t)=2 \psi x y-2 \psi x^{2}+2 x u+\gamma x y
$$

Rearranging equation (11) to conform to the format given in (2) and solving for $u$ [27] results in (12)

$$
\begin{array}{r}
2 \psi x y-2 \psi x^{2}+\gamma x y=-2 x u \\
u=-\psi y-\psi x-0.5 \gamma y
\end{array}
$$

In order for eq. (11) to satisfy the condition for asymptotic stability stated in (6), the feedback control law (12) must be manipulated to render $\dot{V}^{j}(x, y, z, t) \leq 0 \forall(x, y, z) \neq 0$. Thus, we choose

$$
u=-\psi y-0.5 \gamma y
$$

Equation (13) is used to derive the control signal produced by each fuzzy subsystem [28]. In general, we have

$$
u_{i}=-y(\psi+0.5 \gamma), \quad i=1,2, \ldots n, n=9
$$

$n$ is number of fuzzy rules. The conditions for local stability of each fuzzy subsystem are summarized in Table 2.

Table 2. Stability conditions for fuzzy controller

\begin{tabular}{|l|l|}
\hline$u_{4}=-y(\psi+0.5 \gamma)$ & $-80<x<0 ; 0<y \leq 60$ \\
\hline$u_{5}=y(\psi+0.5 \gamma)$ & $0<x \leq 80 ;-5 \leq y<0$ \\
\hline$u_{6}=y(\psi+0.5 \gamma)$ & $-80 \leq x \leq 0 ;-5 \leq y<0$ \\
\hline$u_{7}=-y(\psi+0.5 \gamma)$ & $-10 \leq x \leq 0 ; 0 \leq y \leq 60$ \\
\hline$u_{8}=y(\psi+0.5 \gamma)$ & $-10 \leq x \leq 0 ;-60 \leq y<0$ \\
\hline$u_{9}=y(\psi+0.5 \gamma)$ & $-10 \leq x \leq 0 ;-5 \leq y \leq 0$ \\
\hline
\end{tabular}

\section{SIMULATION RESULTS}

The results of simulation of the closed-loop system using MATLAB software is given in this section. The closed-loop system was simulated for initial conditions $[\mathrm{x}(0), \mathrm{y}(0), \mathrm{z}(0)]=$ $[50,50,-50]$. The controlled trajectories are given in Figure 7. 


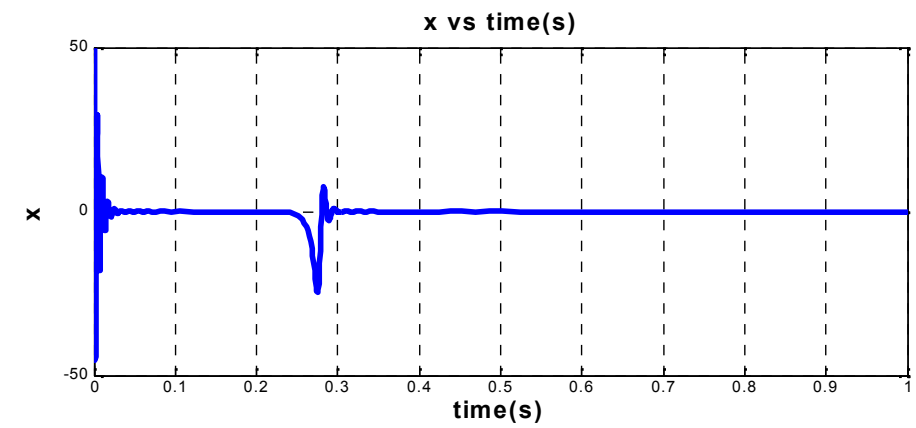

(a)

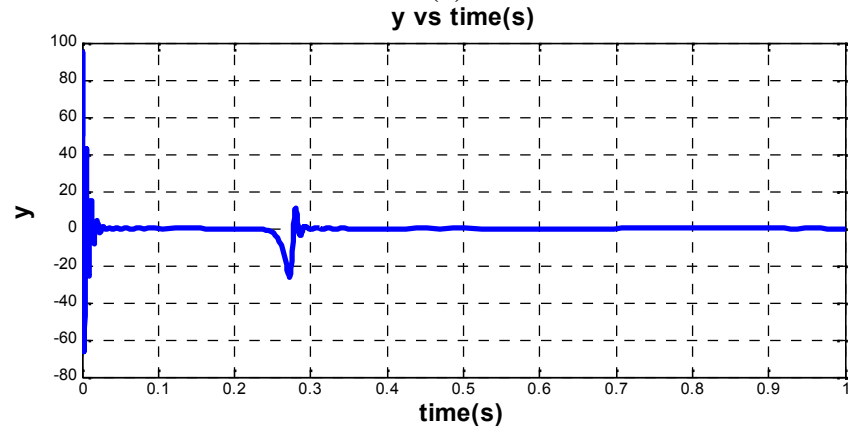

(b)

z vs time(s)

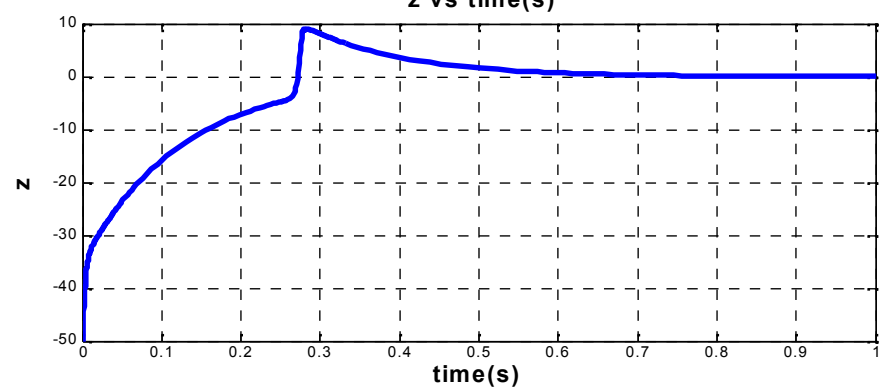

(c)

Figure 7. Controlled trajectories of the hyperchaotic Ai-Yuan-Zhi-Hao system

\section{CONCLUSION}

This paper presents the results of the simulation of a Fuzzy Logic Controller designed to control a hyperchaotic AI-YUAN-ZHI-HAO system via Takagi-Sugeno fuzzy modelling approach. It also presents the results of investigation of the stability bounds necessary for the asymptotic stabilization of the hyperchaotic system based on Lyapunov stability criteria. Simulations results show that the system is hypersensitive to perturbation in its initial conditions. Stability was strongly dependence on the variation of the initial conditions and heuristic tuning of the gains of the controller. For the set of controller gains used in this work, the initial conditions that guarantees asymptotic stability lays within the range of $-50 \leq[\mathrm{x}(0), \mathrm{y}(0), \mathrm{z}(0)] \leq 50$ for the stability conditions tabulated in Table 2 . 
International Journal on Soft Computing, Artificial Intelligence and Applications (IJSCAI), Vol.2, No.4, August 2013

\section{REFERENCES}

[1] A. Kumar \& B.M. Hegde (2012) " Chaos theory: impact on and applications in medicine", Nitte Univ. J. Health Sci., Vol. 2, No. 4, pp 93-99.

[2] R. Robertson \& A. Combs (1995) "Chaos theory in psychology and the life sciences", New Jersey: Lawrence Erlbaum Associates.

[3] D. Guegan (2009) "Chaos in economics and finance", Annual Reviews in Control, Vol. 33, No. 1, pp 89-93.

[4] P.Sooraksa \& K. Klomkarn (2010) "No-CPU chaotic robot: from classroom to commerce", IEEE Circuits and System Magazine, Vol. 10, pp 46-53.

[5] M.J.M. Tavera, M.S. Dutra, E.Y.V. Diaz \& O. Legerke (2009) "Implementation of chaotic behaviour on a fire fighting robot". In: Proc. Int. Conf. Mech. Eng, Gramado, Brazil.

[6] L.S. Martin-Filho \& E.E.N. Macau (2007) "Trajectory planning for surveillance missions of mobile robots". In: Studies in computational intelligence, Springer-Verlag, Berlin, Heidelberg, pp 109-117.

[7] E. Bollt, Y-C. Lai and C. Grebogi (1997) "Coding, channel capacity and noise resistance in communicating with chaos", Phy. Rev. Letts., Vol. 79, No. 19, pp 3787-3790.

[8] Ch. K. Volos, I.M. Kyprianidis \& I.N. Stouboulos (2012) "A chaotic path planning generator for autonomous mobile robots", Robotics and Autonomous Systems, Vol. 60, pp 651-656.

[9] G. Chen \& T. Ueta (1999) " Yet another chaotic attracor",Int.J.Bifurcation Chaos, Vol.9, pp 1465$1466,1999$.

[10] F. Yu \& C. Wang (2013) "Generation of a new three-dimensional autonomous chaotic attractor and its four wing type", Engineering Technology and Applied Science Research, Vol. 3, No. 1, pp 352358.

[11] V. Sundarapandian \& I. Pehlivan (2012) " Analysis, control, synchronization and circuit design of a novel chaotic system", Maths. Comp. Mod., vol. 55, pp 1904-1915.

[12] X. Wang, J. Chen, J-A. Lu \& G. Chen, "A simple yet complex one-parameter family of generalized Lorenz- like systems", retrieved from www.arXiv:1101.4262v3 [nlin.CD] 27 Jan 2011.

[13] A.S. Pikovsky, M.I. Rabinovich \& V.Y. Traktengerts, " Onset of stochasticity in decay confinement of parametric instability", Sov. Phys. JETP, vol.47, pp.715-719, 1978.

[14] K.M. Cuomo, A.V. Oppenheim \& H.S.Strogatz (1993). " Synchronization of Lorenz-based chaotic circuits and application to secure communication", IEEE Transaction on Circuits and Systems-II: Analogue and Digital Processing, Vol. 40, No. 10, pp 626-633.

[15] Y. Nakamura \& A. Sekiguchi (2001) "The chaotic mobile robot", IEEE Trans. Rob. Aut., Vol. 17, No. 6, pp 898-904.

[16] L. Guessas (2006) "Control law based on backstepping design for controlling Lorenz chaotic system", J. Eng. Appl. Sci., Vol.1, No. 4, pp 504-507. 
International Journal on Soft Computing, Artificial Intelligence and Applications (IJSCAI), Vol.2, No.4, August 2013

[17] S. Vaidyanathan (2011) "Adaptive control and synchronization of a highly chaotic attractor", Int. J. Info. Sci. Tech., vol. 1, No.2, pp. 1-11.

[18] B. Rezaie, Z.R.Cherati, M.R.J. Motlagh \& M. Farrokhi (2006) "Stabilization of the Lorenz chaotic equations by fuzzy controllers, Proceedings of the WASET, Vol.15, pp 246-249.

[19] S.Y-Zhong,, Z. G-Zhou \& Q. D-Lian (2006) "Passive control of chaotic system with multiple strange attractors". J. Chinese Phys. Soc., Vol. 15, No.10, pp 2266-2270.

[20] X. Zhang, A. Khadra, D. Li \& D. Yang (2008) "Impulsive stability of chaotic systems represented by T-S model", Chaos, Solit.Fractals, doi:10:1016/j.chaos.2008.07.052.

[21] W. Ai-Yuan \& L. Zhi-Hao (2010) "Dynamic analysis and circuit simulation of a new threedimensional chaotic system", Chin. Phys. B, vol. 19, No. 7, pp 070506-1-6, 2010.

[22] K. Tanaka \& H.O. Wang (2001) "Fuzzy Control Systems Design and Analysis: A Linear Matrix Inequality Approach", New York: John Wiley

[23] T. Takagi, M. Sugeno, "Fuzzy identification of systems and its application to modeling and control", IEEE Trans.Man. Cybernet., 15:116-132, 1985

[24] K.-Y. Lian, C.-S. Chiu, T.-S. Chiang \& P. Liu (2001) "LMI-based fuzzy chaotic synchronization and communications", IEEE Trans. Fuzzy Syst. Vol. 9, No.4.

[25] L.K. Wong, F.H.F. Leung, P.K.S. Tam, " Lyapunov function-based design of fuzzy controllers and its application on combining controllers", IEEE Trans Ind. Electron. 45(3):502-509, 1998

[26] L.K. Wong, F.H.F. Leung, P.K.S. Tam, "Lyapunov function-based design of heuristic fuzzy logic controllers", IEEE Int. Conf. Fuz. Syst., 1:281-285, 1997

[27] L.K. Wong, F.H.F. Leung \& P.K.S. Tam, "An improved Lyapunov function-based stability analysis method for fuzzy logic control systems", Electron. Lett. Vol. 36, No. 12, pp 1085-1096.

[28] R.E. Precup, M.L. Tomescu \& S. Preitl, "Stabilization of Lorenz system using Fuzzy controllers", Int. J.Comp. Commun. Contr., Vol. 2, No.3, pp279-287.

\section{Author}

Edwin A. Umoh received the B. Eng (Electrical/Electronics) and M. Eng (Electronics) degrees in 1995 and 2011 from Abubakar Tafawa Balewa University, Bauchi, Nigeria. He is currently a Senior Lecturer in the Department of Electrical Engineering Technology, Federal Polytechnic, Kaura Namoda, Nigeria. Engr Umoh is a member of the IEEE Control System Society and IEEE Computational Intelligence Society. His research interests are in chaos control, fuzzy modelling and illumination engineering.

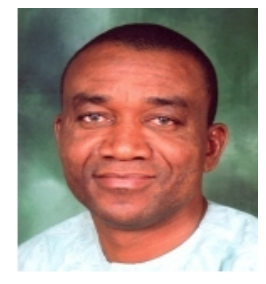

